\title{
CURRENT ACHIEVEMENTS IN TIME TRANSFER
}

\author{
G. Petit and C. Thomas
}

\begin{abstract}
Since early eighties, the introduction of new methods for distant time transfer has led to a major improvement in the precision, accuracy, and coverage of world-wide time metrology. All of these methods are based on the use of satellites. Communication between ground stations and satellites is effected through microwave or optical signals according to a one-way or twoway mode.
\end{abstract}

The one-way mode and the corresponding time transfer methods can be operated with two global satellite navigation systems, first designed for positioning. There are:

* The American Global Positioning System, GPS, composed of 24 satellites equipped with caesium clocks. At present days, the GPS is operational as well for what concerns the satellite constellation as for what concerns station equipment. It is a military system which denies its full potentiality to civil users. However, it is widely used for dissemination of the time scale GPS time, and for clock synchronization by the common-view method. It leads to uncertainties in ground clock synchronization of order 3,6 ns $(1 \sigma)$ for short-distance links ( $\leq 1000 \mathrm{~km}$ ), and, if data is corrected for measured ionospheric delays and post-processed precise satellite ephemerides, of order $5 \mathrm{~ns}(1 \sigma)$ for long-distance links ( $\geq 5000 \mathrm{~km})$ [1].

* The Russian GLObal NAvigation Satellite System (GLONASS) is equivalent to GPS. At present days, the GLONASS is not operational: only few satellites are launched and commercial time receivers are not yet available. A comparative experiment between GPS and GLONASS common-view time transfers showed that the GLONASS can provide continental time transfer at a level of several tens of nanoseconds, a performance which could be further improved by the use of ionospheric and tropospheric models [2].

The two-way method has already been successfully tested using geostationary satellites. The first most important results have been obtained in 1990 using the geostationary European communication satellite EUTELSAT. It led to a precision of one single measurement of order several hundreds of picoseconds, and to an uncertainty of $1,7 \mathrm{~ns}(1 \sigma)$ for the link between the Observatoire de la Côte d'Azur, OCA, Grasse (France), and the Technical University of Graz, TUG, (Austria) [3]. This required, on site, stations for emission and reception of microwave signals in the telecom band, as well as a satellite channel for signal repetition on board.

The two-way mode can also be operated using light rather than microwave signals. This call for the operation of an Earth laser sufficiently powerful for shooting onto a satellite and for a light retro-reflector in the satellite payload. This is the LAser Synchronization from Satellite Orbit (LASSO), already successfully experienced. This method requires heavy ground equipment, and also particular good weather conditions on each stations in order to avoid light dispersion. It is thus not an operational method. Results have been obtained between Texas and France, but no accuracy evaluation has yet been possible. The precision of one-single time difference measurement is of order $50 \mathrm{ps}$ [4]. 
Possible improvements are yet possible for each of these methods, and may become necessary, in particular for comparison of future clocks designed for frequency accuracies of some parts in $10^{16}$.

\section{$\underline{\text { References }}$}

1. Lewandowski W., PetTT G., and Thomas C., Precision and Accuracy of GPS Time Transfer, IEEE Trans. Inst. Meas., 42, 2, 1993, 474-479.

2. Daly P., Koshel yaevsky N.B., Lewandowski W., Petit G., and Thomas C., Comparison of GLONASS and GPS Time Transfers, Metrologia, 30, 1993, 89-94.

3. KirChNeR D., RESSleR H., Grudler P., Baumont F., VeIllet C., LEWANDOWSKi W., HANSON W., KLEPCZYNSKI W., and UHRICH P., Comparison of GPS Common-view and Twoway Satellite Time Transfer Over a Baseline of 800 km, Metrologia, 30, 1993, 183-192.

4. VEILLET C., and FRDELANCE P., Time transfer between USA and France through LASSO, Proc. 7th EFTF, 1993, 637-640. 